\title{
Efficient ICT for efficient Smart Grids
}

\author{
Gerard J.M. Smit, University of Twente, Enschede, the Netherlands \\ contact: g.j.m.smit@utwente.nl
}

\begin{abstract}
In this extended abstract the need for efficient and reliable ICT is discussed. Efficiency of ICT not only deals with energy-efficient ICT hardware, but also deals with efficient algorithms, efficient design methods, efficient networking infrastructures etc. Efficient and reliable ICT is a prerequisite for efficient Smart Grids. Unfortunately, efficiency and reliability have not always received the proper attention in the ICT domain in the past.
\end{abstract}

\section{A. Introduction}

Although much of the worldwide increase in electricity consumption is attributed to ICT, the same ICT technology can also be used to reduce the worldwide energy consumption. Efficient ICT systems can, for example, be used to control the consumption, production, transportation, and storage of electricity (Smart Grids). Next ICT systems can be used to control and schedule large electricity consuming and producing appliances in an optimal way. Therefore, ICT plays a key role in reducing the energy intensity of the economy, thus helping to decouple growth from energy consumption and creating new opportunities while saving the environment.

Research in using ICT for reducing energy consumption may include issues such as e.g.

- flexible ICT systems for (decentralized) electricity management

- technologies and tools for dependable ICT systems that continue to operate when elements fail in the electricity network (Smart Grids),

- versatile multi-purpose energy-efficient ICT systems and communication infrastructures which can handle a wide range of (future) services to safeguard sustainability, and

- energy-aware systems which integrate real-time information from (wireless) sensor networks and external information sources such as weather forecasts.

In March 2007, the European Council agreed on an action plan on European energy policy. This action plan is known as the EU 20-20-20 plan. It sets new, binding targets for the reduction of greenhouse gas emissions from energy consumption. These will be met through increasing the use of renewables to $20 \%$ of total energy consumption by 2020 , mostly via highly decentralized 'green' power generators (like from wind, biomass, or solar energy; or from micro-CHPs or energy storage systems) forming Virtual Power Plants. Furthermore, improving energy efficiency should ensure that the total energy usage is $20 \%$ lower than current projections for

G.J.M. Smit is professor of the Computer Architecture for Embedded Systems (CAES) group within the Department of Electrical Engineering, Mathematics and Computer Science, University of Twente, P.O. Box 217, 7500 AE, Enschede, The Netherlands
2020, through reduction of consumption and of transmission losses as well as improvement of energy-conversion efficiency.

The energy technologies in use today were mostly designed for a different era based on centralised energy generation and economies of scale. Today the energy companies are facing a transition to address the goals of the new EU energy policy. This will require new energy and ICT infrastructures. The driving forces for this transition are the security of energy supply and environmental concerns. ICT will be an enabler in this process and will lead to the creation of new energy markets, decentralized control, and higher system quality, efficiency and reliability.

According to a recent report 2020 [1] of the Climate Group and McKinsey, ICT is a key player in the battle against climate change and offers the possibility of $7.8 \mathrm{Gt}$ reduction of $\mathrm{CO}_{2}$ emission in 2020. A recent annex of this report calculates a saving of 140-240 billion dollar in the US by reducing the energy consumption due to smart usage of ICT systems. Also the European Commission is stressing the importance of ICT for energy reduction and sustainability and invests in research in this area [2]. The plans have amongst others resulted in a number of research programmes combining ICT with Energy. For example the EU-PPP programme on Energy Efficient Buildings and the EU-FP7 calls for ICT for Energy Efficiency, ICT for environmental services and climate change adaptation, novel ICT solutions for Smart Electricity Distribution Networks, ICT for energy-efficient buildings and spaces of public use and ICT for energy-positive neighbourhoods.

\section{B. Efficient ICT}

Energy consumption is one of the main concerns of todays ICT hardware and software designers and systems integrators. Regarding energy-efficiency the first type of devices people usually think of are portable devices as these devices rely on batteries and therefore the functionality is strictly limited by the energy consumption. However, for high performance computing there is also an increasing need for energy-efficient architectures to reduce the cost for cooling and packaging. Today also the performance of many ICT systems is limited by energy dissipation and electricity supply. In addition to that there are environmental concerns that urge for more efficient architectures in particular for systems that run 24/7 (24 hours per day 7 days per week) such as wireless base stations, servers parks, data centres and search engines (e.g. Google has an estimated server park of one million servers that run 24/7). Energy reduction can be done at several layers: reduce energy for processing and storage of data, reduce energy for communication, operating systems and software.

One important aspect in energy reduction is locality of reference. This means that data that a processing element 
frequently needs should be located close to the processing element. For example it is quite inefficient when sensor data is brought to a central location, where the processing is done and brought back to the actuators, that are often located close to the sensors, where actuation is done. When wireless networks are needed to transport this data, often more energy is spent in data communication compared to the energy in the central processing. This means that processing should be done close to sensors whenever possible. In our opinion the cloud computing paradigm is less applicable for Smart Grids, because data is distributed over many devices scattered over a large area and the processing is rather simple. However, cloud computing can be used for high-level control and optimisation of energy streams.

\section{Energy Management}

In a few years from now a large percentage of the electricity will be generated by decentralized micro-generators, (e.g. micro-CHPs and solar cells). Micro generation systems generate electricity at the kilowatt level which will allow the units to be installed in an individual home and connected directly with the domestic heating and electrical systems, which by definition entails very high efficiency (up to 90\%) in usage of primary energy. Also, it allows for delivery of electricity to the power grid, where the distance to its consumer may be limited, e.g. in the same city or neighbourhood. The micro-generation concept has the additional benefit of reduced distribution and transmission losses, delivering significant advantages in terms of overall efficiency relative to centrally generated power. This concept offers an attractive option in the reduction of primary energy consumption and therefore carbon $\left(\mathrm{CO}_{2}\right)$ emissions. On the long run we will rely on renewable energies.

Renewable energies are generally based on a large number of even smaller sources of power, producing power much closer to the location in which it is used. Some installations, such as those producing heat, can only supply users in their immediate area, while equipment which produces electricity, such as windmills, biomass systems, or photovoltaic roof panels, can be used to supply to electricity networks.

The introduction of renewable energy requires the development of new ICT systems for obtaining energy efficiency and meeting grid requirements, while maintaining high standards of comfort, quality and reliability of services as well as connectivity. In addition, the future availability of storage devices (such as batteries in electric cars) opens up new perspectives and constraints, both from the sides of storage and supply (to the grid), as well as demand (from the grid). The demand for electricity in a large network varies dynamically as millions of users continuously switch on and off independently their equipment. The wholesale price of electricity, paid by distributors to (large scale) generators, also rises and falls with demand. A similar approach could be applied to massive amounts of small-scale generators, seen as a Virtual Power Plant (VPP).

An intelligent system employed by the operator of a smallscale generation resource, such as photovoltaic panels on a house, or a fuel-cell, would be able to recognise when surplus energy can best be sold on the market, and when it should be used to heat water, for example. Similarly, continuous agreements for supplies during short or long durations, involving e.g. prices, timing, and quantities could be made between network operators and small-scale suppliers.

ICT provides the means to manage the operation of millions of small-scale electricity generation appliances, both on behalf of their owners and for the networks into which they feed. ICT tools can monitor a range of variables and ensure that both individuals and the network as a whole gain maximum efficiency from the energy generation capacity available. Development of such ICT systems will therefore fuel the installation of new and renewable-energy-based generation capacity, through maximising the return on investment so making it more affordable. In this way, it can more quickly meet its goals in the field of stable electricity supply, energy efficiency and emissions reduction, and provide consumers with higher quality energy supplies.

The electricity grid has to cope with the variability of the future generators and consumers, many of which are still unknown. In principle, the smart grid is an upgrade of 20th century power grids, which generally "broadcast" power from a few central power generators to a large number of users, to instead be capable of routing power in more optimal ways to respond to a very wide range of conditions. The conditions, to which a smart grid can respond, may occur anywhere in the power generation, distribution and demand chain. Events may occur generally in the environment (clouds blocking the sun and reducing the amount of solar power, a very hot day), commercially in the power supply market (prices to meet a high peak demand exceeding one dollar per kilowatthour), locally on the distribution grid (MV transformer failures requiring a temporary shutdown of one distribution line) or in the home (someone leaving for work, putting various devices into hibernation), which motivate a change to its power flow. In addition, several constraints are induced by the power grid itself as well as the devices connected to it.

One of the problems Smart Grids are facing with is that not all decentralized micro-generation systems have predictable electricity generation patterns. For example solar cells are dependent on the time of the day and the amount of sunlight. Wind energy is only available when there is wind and microCHP systems only produce electricity when there is a heat demand. As a consequence, decentralized systems sometimes produce electricity when there is no need for it locally.

A solution to these problems may be to transform domestic customers from static consumers into active participants in the production process. Consumer participation can be achieved through the development of new (domestic) devices with controllable load, micro-generation and domestic energy storage of both heat and electricity. These devices have potential to shift electricity consumption in time without harming the comfort of the residents.

\section{3 step control methodology}

Examples of devices with optimization potential are (smart) freezers and fridges, which can adjust their cooling cycles to 
shift their electricity load and (electrical car) batteries that can temporarily store excess electricity. To exploit this optimization potential on a large scale, a global control methodology is required [3]. The above-mentioned challenges and optimization potential are studied and a control methodology is derived. This control methodology aims:

- to achieve a more efficient use of the generated electricity of existing power plants,

- to facilitate the large scale introduction of renewable sources and

- to allow large scale introduction of new technologies for production, consumption and storage while at the same time maintaining grid stability and ensuring a reliable and affordable supply.

The control methodology uses mathematical optimization techniques and a combination of prediction, offline global planning based on the predictions and online realtime control based on the global planning. The base of the control methodology is:

- using local information,

- communication using multiple levels and

- scalability.

The goal of the control methodology is to work towards (global) objectives and the performance of the control methodology is measured to the extent to which the objectives are reached. Based on the above considerations, the proposed control methodology uses three steps and is split up into a local and a global part:

1) predictions on a device level to be able to predict the overall result,

2) planning to estimate the energy streams in the building and the grid and

3) real-time control to respond on changes (e.g. fluctuations in renewable generation) and work around predictions errors.

In the first step, the energy usage or production for every individual building in the grid is predicted on a device level. In the second step, the predictions of the individual buildings are aggregated and a global planning is made in an iterative and hierarchical way. The result of this planning is an energy profile. In the last step, a local scheduler in every building schedules the devices in real-time, using steering signals determined by the global planning as an input.

Because of scalability reasons, the global planning has a hierarchical structure and can aggregate data and planning on different levels, e.g. within a neighbourhood or city. The level on which the planning is determined and, thus, the highest level of the global controller determines the scope (e.g. a local scope or a neighbourhood scope). Especially the three steps and the global planning differ from the control methodologies described in literature. Furthermore, the control methodology is not agent based and uses other mathematical optimization methods or heuristics than the control methodologies described above.

Due to the predictions and planning on beforehand, the predictability of the global electricity streams is improved. The combination of planning (aggregated knowledge on higher levels) and mathematical optimization result in better dependability and combination of planning and real-time control improves the control over unexpected events. Furthermore, the amount of communication can be limited due to the hierarchical structure. Finally, the requirements on the communication medium are low since the local controller can work independently and a lot of information can be sent on beforehand without high latency requirements.

Simulations show that the control methodology can optimize the energy flows and can control the operation of the domestic devices in an economic manner without discomfort for the residents. The prediction and planning step preceding the realtime control improves the results of the control methodology: much more optimization potential can be exploited and the results are more predictable and dependable.

The challenging issues on the non-technical side include regulatory regimes, business models, user psychology (including awareness) and privacy, governance and social dynamics. Widespread distributed generation requires completely new regulation since the traditional roles of producers and consumers are about to change. For the same reason, new business models are needed. It might intervene quite substantially in the daily energy routines of households with severe consequences for routines and privacy. Finally, it might initiate new social communities with new functions and activities. Some challenges are:

- Behaviour of end-users

- Privacy and data protection

- Economics and transactions: who pays for what

- Legislation and regulation issues

\section{E. Conclusion}

ICT provides the enabling technology to realise the EU 20-20-20 goals. The energy technologies in use today were mostly designed for a different era based on centralised energy generation and economies of scale. Today we are facing a transition to address the goals of the new EU energy policy. This will require new energy and ICT infrastructures. The driving forces for this transition are the security of energy supply and environmental concerns. ICT will lead to the creation of new energy markets, decentralized control, and higher system quality, efficiency and reliability. An important aspect is that for future energy systems prediction and planning is required.

\section{REFERENCES}

[1] The Climate Group, Global e-Sustainability Initiative (GeSI), "Smart 2020: Enabling the low carbon economy in the information age," 2008 http://www.smart2020.org/.

[2] Commission of the European Communities, "Mobilising ict to facilitate the transition to an energy-efficient, low-carbon economy," 2009.

[3] A. Molderink, V. Bakker, M. G. C. Bosman, J. L. Hurink, and G. J. M. Smit, "Management and control of domestic smart grid technology," IEEE Transactions on Smart Grid, vol. 1, no. 2, pp. 109-119, 2010. 